\title{
Uso da simulação realística como estratégia de ensino para os cursos de graduação em enfermagem
}

\section{Use of realistic simulation as a teaching strategy for undergraduate nursing courses}

\author{
Thiago da Silva ${ }^{1 *}$, Amaranta Rangel Ramos ${ }^{1}$, Alexander de Quadros $^{1}$
}

\begin{abstract}
RESUMO
Trata-se de uma pesquisa qualitativa, descritiva exploratória do tipo revisão integrativa da literatura. Teve por objetivo analisar as publicações sobre prática clínica simulada, ou simulação realística, aplicada nos cursos de graduação em Enfermagem como estratégia de ensino e aprendizagem para o desenvolvimento das competências recomendados pelas Diretrizes Curriculares Nacionais (DCNs). As bases de dados exploradas foram LILACS, PUBMED, COCHRANE e SCIELO, abrangendo o período de 2010 - 2020. A coleta de dados realizou-se por meio de um instrumento estruturado utilizando o modelo Preferred Reporting Items for Systematic Reviews and Meta-Analyses - PRISMA. Após a leitura exploratória dos resumos e a aplicação dos critérios de inclusão e exclusão pré-estabelecidos, foram selecionadas 18 publicações, cujo conteúdo foi lido integralmente e destas 14 publicações compuseram estudo final apresentados por meio de um quadro sinóptico. Os resultados evidenciaram que o uso da simulação realista como estratégia de ensino tem sido de interesse nacional e internacional, aproximando os estudantes e profissionais aos contextos reais simulados, oferecendo completa segurança para os envolvidos, buscando desenvolver não somente habilidades técnicas, como também o gerenciamento de crises, liderança, trabalho em equipe, raciocínio clínico em situações críticas ou que possam provocar prejuízos ao paciente real, sendo uma ferramenta poderosa de aprendizagem. A discussão dos principais achados dessas publicações e seus desfechos finais estão sendo compilados em um artigo científico, visando contribuir com a produção de conhecimento para os cursos de graduação em enfermagem.
\end{abstract}

Palavras-chave: Enfermagem; Simulação; Simulação de Paciente; Educação em Enfermagem; Aprendizado Ativo.

\section{ABSTRACT}

This is a qualitative, descriptive exploratory research of the integrative literature review type. It aimed to analyze publications on simulated clinical practice, or realistic simulation, applied in undergraduate nursing courses as a teaching and learning strategy for the development of skills recommended by the National Curriculum Guidelines (DCNs). The databases explored were LILACS, PUBMED, COCHRANE and SCIELO, covering the period 2010-2020. Data collection was carried out through a structured instrument, using the Preferred Reporting Items model for Systematic Reviews and Meta-Analyses - PRISMA. After the exploratory reading of the abstracts and the application of pre-established inclusion and exclusion criteria, 18 publications were selected, whose content was read in full,14 publications made up the final study presented through a synoptic table. The results showed that the use of realistic simulation as a teaching

\footnotetext{
${ }^{1}$ Faculdade Meridional IMED. *thiago.silva@imed.edu.br
} 
strategy has been of national and international interest, bringing students and professionals closer to the real simulated contexts, offering complete security for those involved, seeking to develop not only technical skills, but also the management of crises, leadership, teamwork, clinical reasoning in critical situations or that may harm the real patient, being a powerful learning tool. The discussion of the main findings of these publications and their final outcomes are being compiled in a scientific article, aiming to contribute to the production of knowledge for undergraduate nursing courses.

Keywords: Nursing; Simulation; Pacient simulation; Nursin education; activ learning

\section{INTRODUÇÃO}

Na educação, decidir e definir os objetivos de aprendizagem significa estruturar, de forma consciente, o processo educacional de modo a oportunizar mudanças de pensamentos, ações e condutas dos estudantes(BARISTA, 2013). O uso da simulação realística como estratégia de ensino nos cursos de graduação em enfermagem vem sendo implementada de forma significativa nos últimos anos, como uma metodologia inovadora que permite ao aluno desenvolver os domínios de aprendizagem cognitivo, afetivo e psicomotor (DE OLIVEIRA COSTA et al., 2018).

Este framework da taxonomia aliada a simulação, permite a organização de um sistema pré-determinado para discussões, análises e/ou recuperação de informação. O uso da simulação realística aliada a taxonomia de Blomm, permite ao docente definir objetivos de aprendizagem de forma clara e objetiva, dentro de uma pirâmide aplicando desde os níveis mais básicas quanto aos mais elevados no processo de aprendizagem, contribuindo na formação deste futuro enfermeiro para uma tomada de decisão mais precisa e Segura (DE OLIVEIRA COSTA et al., 2018; FERRAZ e BELHOT, 2010; FERREIRA; GUEDES; OLIVEIRA, 2018).

A Simulação Realística, se constitui em uma metodologia que oferece aos estudantes da saúde a possibilidade de realizar de maneira segura e controladas, uma prática análoga a que realizará na prática profissional, promovendo desta forma, uma assistência segura, contribuindo para as dimensões relacionadas a segurança do paciente (FERREIRA; GUEDES; OLIVEIRA, 2018).

O cenário em simulação realística em saúde é uma parte integrante e fundamental para o planejamento e organização dos cursos de formação profissional, capacitação e 
treinamento de profissionais de saúde e atuação interprofessional (VILELLA; LEITE; NASSAR, 2018). A Diretriz Curricular Nacional para a área de Enfermagem (DCN/ENF) estabelece normas obrigatórias que regulamentam a formação do enfermeiro na graduação, proporcionando uma direção única e estabelecendo o perfil do egresso independente da universidade de formação. A DCN foi estabelecida pela Resolução CNE/CES no 03/2001, após um movimento de articulação e diálogo com profissionais enfermeiros, professores de enfermagem, Cofen e ABEN. Essa diretriz ressalta a importância da construção de Projetos Político-Pedagógicos (PPP), que contemplam as dimensões necessárias à formação dos enfermeiros para uma formação generalista, crítica e reflexiva, capaz de atuar em todos os níveis de atenção em saúde no Brasil (GONZALES e ALMEIDA, 2010).

No contexto atual onde o virtual impera, muitos são os desafios para acompanhar e aprimorar os métodos de ensino utilizados com o propósito de alcançar uma formação profissional deste futuro enfermeiro mais crítico, humanista e reflexivo. A metodologia com simulação realística transporta o discente a cenários próximos do real, onde se é permitido errar, refazer procedimentos, discutir intervenções e realizar um atendimento ao paciente sem riscos, possibilitando, portanto, uma formação mais robusta ao consolidar teoria e prática simultaneamente (NEGRI et al., 2017)

Um estudo da África do Sul, realizado com 8 estudantes da graduação em Enfermagem, revelou que os alunos percebem a simulação com pacientes-padrão como uma ferramenta que preenche a lacuna entre a teoria e a prática podendo, a partir da simulação, aplicar os conhecimentos teóricos adquiridos. Além disso, o estudo identificou que os alunos identificam suas próprias potencialidades e fragilidades a partir da reflexão dos cenários vivenciados (KELLY, 2014).

A prática simulada, no ensino de Enfermagem, é uma realidade em Portugal e tem contribuído para a melhoria do processo formativo, do desenvolvimento de autoconfiança, autonomia e satisfação. No Brasil, os estudos sobre a temática ainda são incipientes e ainda carece de produção de evidências. Confirmando este fato, até julho de 2017, apenas 6 ensaios clínicos estavam cadastrados na plataforma de Registro Brasileiro de Ensaios Clínicos (COSTA et al., 2017).

Hoje, é indispensável considerar a utilização de equipamentos como manequins e simuladores, dentre outras tecnologias, no processo de formação de profissionais de 
saúde. Pois, aprender e praticar técnicas e ou procedimentos em pacientes em situação de vulnerabilidade passa a ser uma estratégia primitiva, inadequada e eticamente injustificável, sempre que o treino prévio for possível no contexto simulado (FERREIRA; GUEDES; OLIVEIRA, 2018; KELLY, 2014).

A experiência clínica simulada pode oferecer maior suporte ao aprendizado clínico, direcionando as atividades simuladas para as necessidades de aprendizado específicas, bem como na avaliação de desempenho. A mudança na formação superior dos novos profissionais de saúde é necessária, valorizada e estimulada, mas por que é tão difícil mudar? Quais são as estratégias usadas na tentativa de implantar mudanças locorregionais?

Para responder essas inquietações se elencou como objetivo geral desta pesquisa, analisar as publicações sobre prática clínica simulada aplicadas nos cursos de graduação em enfermagem. O ensinar e o aprender formam uma unidade que delimita, culturalmente, o campo de constituição do indivíduo. São processos indissociáveis em que o professor participa ativamente da constituição de processos psíquicos do estudante. O professor deve, portanto, se responsabilizar, em parceria com o estudante, pelo processo de aprendizagem. Vygotsky demonstra a unidade do processo ensino aprendizagem em sua abordagem histórico-cultural e, neste processo, a cultura e o indivíduo se constituem mutuamente (TUNES; TACCA; BARTHOLO JUNIOR, 2005).

Observa-se na prática cotidiana o distanciamento entre o processo de ensino aprendizagem e os processos de avaliação nas instituições de ensino, para sanar essas dificuldades, é necessário a implementação de novas tecnologias como potenciais estratégias para modernização do ensino. No entanto, deve-se considerar que apenas a utilização da tecnologia não é a garantia de uma melhor aprendizagem havendo a necessidade de serem desenvolvidas ações pedagógicas que possibilitem um fazer crítico vinculado a realidade, construindo na autonomia e na cooperação dos estudantes.

As tecnologias educacionais digitais estão sendo utilizadas cada vez mais na área da saúde colaborando na diversificação e flexibilização das atividades, possibilitando que o estudante acesse os conteúdos em tempo e local que desejar, além de proporcionar a interação entre os estudantes que vai além do espaço físico da sala de aula.

A utilização da metodologia simulação realística é de grande importância na formação do graduando, já que ela desenvolve a formação do pensamento crítico, reflexão 
e estimula á pró-atividade sendo uma ferramenta integradora da teoria e da prática, permitindo ao aluno desenvolver a capacidade de resolver problemas. A simulação na educação dos profissionais de saúde dá aos estudantes a oportunidade de desenvolverem habilidades variadas e competências necessárias em ambiente controlado e protegido. Permite erros e crescimento profissional, sem arriscar a segurança do paciente (FERREIRA; GUEDES; OLIVEIRA, 2018).

A última etapa da simulação permite uma discussão reflexiva (debriefing) sobre a situação ocorrida, da aprendizagem e das decisões tomadas, estimulando o pensamento crítico e reflexivo do estudante, consolidando os saberes. O debriefing é uma ferramenta educacional com base nos princípios da teoria da aprendizagem de adultos que faz uso de um evento simulado (ou real), a fim de gerar uma discussão referente à vivência no cenário (SOUZA et al., 2017).

O diálogo, possibilita interações sociais por meio da palavra e do próprio ato de pronunciá-la, o diálogo não se restringe apenas a linguagem falada, a relação estabelecida no encontro entre aquele que cuida e o cliente é mediada por um espaço intersubjetivo que permite a comunicação e conduz a interação entre eles. Esse encontro implica, também, em construção de conhecimento a partir de um sistema de diferenças e em um compromisso entre os sujeitos para entender tais diferenças. Para isso, a aplicação da metodologia Simulação realística, é vista como um método efetivo e inovador que amplia as relações entre a teoria e a prática do corpo discente em ambiente seguro, oferecendo melhores oportunidades de aprendizagem e treinamento, contribuindo para a formação professional (FERREIRA; GUEDES; OLIVEIRA, 2018).

Fazendo um paralelo entre a simulação realística e os tipos de memória, está metodologia ativa a memória episódica que refere-se onde guardamos toda a nossa experiência de vida é a principal responsável por novas aprendizagens e principalmente a memória processual, onde guardamos, as aprendizagens mais ou menos automáticas, fugindo a consciência (TIEPPO; FONSECA, 2020).

O debriefing é uma etapa indispensável em qualquer processo de ensino simulado. É somente por meio dele que o autoconhecimento é promovido e a aprendizagem se torna de fato significativa. Neste sentido, é preciso concordar que sua prática sistematizada e com a utilização de guidelines favoreça o importante crescimento ao aluno (SOUZA et al., 2017). 
O objetivo geral desta pesquisa foi analisar as publicações sobre prática clínica simulada aplicadas nos cursos de graduação em Enfermagem como estratégia de ensino e aprendizagem para o desenvolvimento das competências recomendados pelas Diretrizes Curriculares Nacionais (DCNs).

\section{MÉTODO}

O método escolhido foi uma revisão integrativa de literatura, técnica de pesquisa que reúne e sintetiza publicações relevantes sobre um delimitado tema ou questão, de modo sistêmico e ordenado, contribuindo para o aprofundamento do conhecimento do tema investigado, possibilitando elaborar conclusões a respeito de uma área particular de estudo. Por ser uma pesquisa de revisão dispensa a aprovação do comitê de ética.

Para a realização desta revisão, foram utilizadas as seguintes fases: identificação do tema e definição da questão norteadora; estratégia de busca e seleção da literatura; categorização, avaliação e análise dos estudos; e apresentação de texto modelo de texto modelo de texto modelo de texto (MENDES; SILVEIRA; GALVAO, 2008).

A questão norteadora desta pesquisa será utilizada a estratégia PICO, que representa um acrônimo para Paciente ou problema, Intervenção, Controle ou comparação e "Outcomes" desfecho (SANTOS; PIMENTA; NOBRE, 2007). Esta revisão foi realizada nas seguintes bases de dados indexadas: Literatura Latino-americana e do Caribe em Ciências da Saúde - LILACS, National Library of Medicine - PUBMED, Scientific Electronic Library Online - SCIELO e The Cochrane Library - COCHRANE, pela combinação dos descritores de busca com a utilização do conector booleano AND, todos os termos em inglês.

O levantamento bibliográfico ocorreu no mês de Maio de 2020 e a análise do material coletado entre Maio e Junho de 2020. Os critérios de inclusão estabelecidos para esta pesquisa foram: artigos acadêmicos, publicados em periódicos com resumo e texto completo, disponíveis nos idiomas português, inglês ou espanhol, e que estiverem adotado o método empírico de investigação do tema em análise, correspondente aos últimos 10 anos 2010-2020.

Da busca nas bases de dados foram encontradas 9.354 publicações na LILACS, 95 artigos na SCIELO, 4 na COCHRANE e 507 na base da PUBMED, totalizando 9.960 
artigos que foram submetidos a leitura dos títulos das publicações e dos descritores, permanecendo 314 artigos. Destas produções, 186 estavam repetidos em 2 bases de dados, sendo 94 artigos submetidos à leitura dos resumos.

Após a leitura exploratória dos resumos e a aplicação dos critérios de inclusão e exclusão pré-estabelecidos, foram selecionadas 18 publicações, cujo conteúdo foi lido integralmente. Para a apresentação das etapas de seleção dos artigos será utilizado o modelo Preferred Reporting Items for Systematic Reviews and Meta-Analyses PRISMA (GALVÃO; PANSANI; HARRAD, 2015).

Figura 1: Fluxo do PRISMA ${ }^{(16)}$
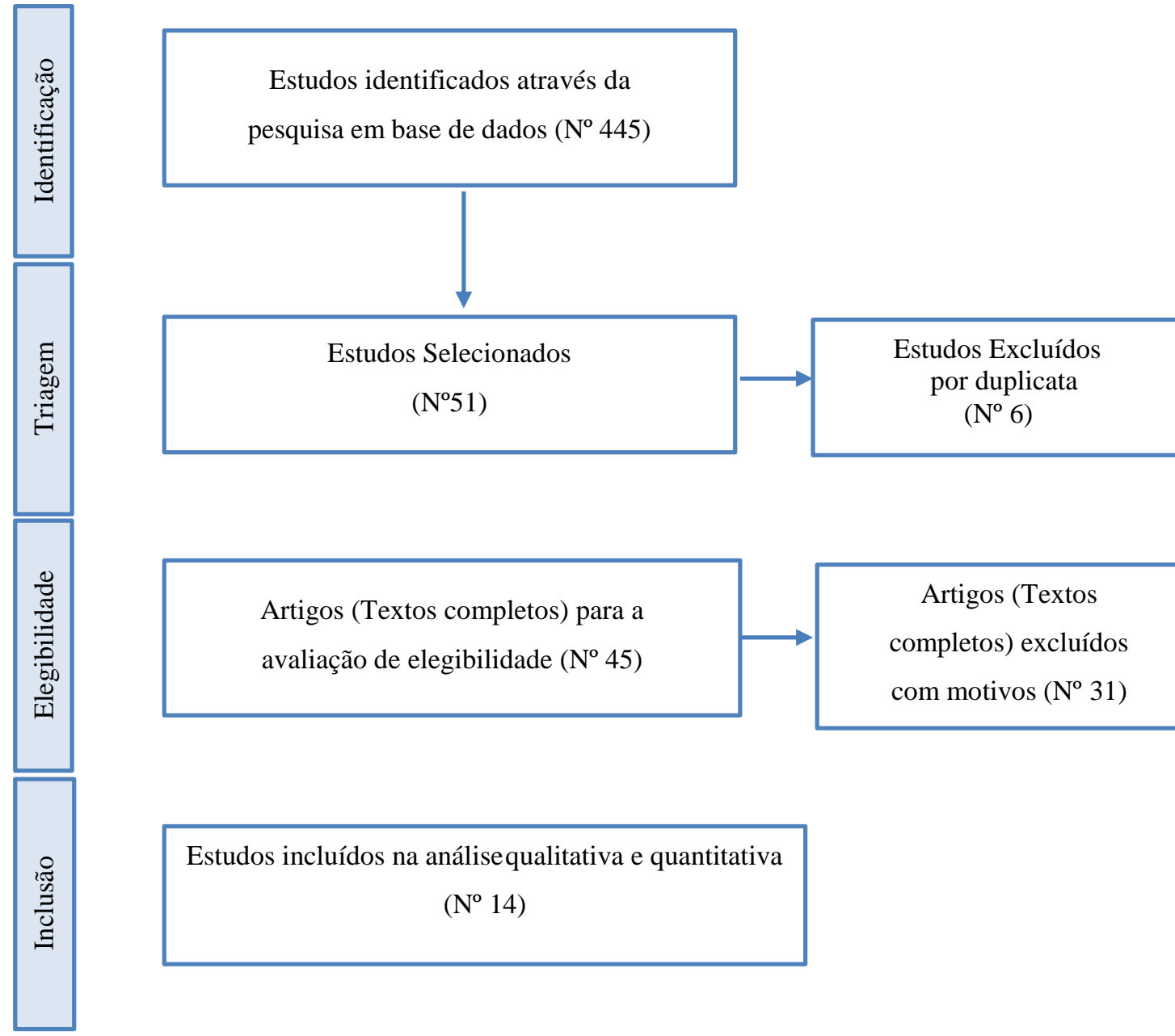

Estudos incluídos na análisequalitativa e quantitativa $\left(\mathrm{N}^{\circ} 14\right)$

Para coleta dos dados, utilizou-se um instrumento detalhado (Quadro 1), contendo: título, autores, ano de publicação, periódico, metodologia e nível de evidência. A análise e síntese dos artigos foram obtidas de forma descritiva, para avaliação do nível de 
evidência dos estudos sendo utilizada a escala de Rating for thehierarchyof Evidence for Intervention/ Treatment Questions (BURNS; ROHRICH; CHUNG, 2011).

Figura 2: Rating for the hierarchy of Evidence for Intervention/ Treatment

Questions

\begin{tabular}{|l|l|}
\hline Nível I & Evidências revisões sistemáticas/meta-análise EC e randomizados. \\
\hline Nível II & Evidências de ensaios clínicos randomizados. \\
\hline Nível III & Evidências de ensaios clínicos sem randomização. \\
\hline Nível IV & Evidências de caso-controle e estudos de coorte. \\
\hline Nível V & Evidências de revisões sistemáticas de estudos descritivos e qualitativos. \\
\hline Nível VI & Evidências de estudos descritivos ou qualitativos. \\
\hline Nível VII & Evidências de opinião de autoridades/relatórios de experts. \\
\hline
\end{tabular}

\section{RESULTADOS}

Foram selecionadas 14 publicações, cujo idioma predominante foi o português 10 (71\%), estudos no Brasil e 4 (29\%) nos EUA. O Quadro 1 apresenta o perfil dos estudos da amostra. Observou-se uma maior prevalência de estudos com nível de evidência VI, 11 (79\%), seguido de nível II, 2 (14\%) e nível VII 1(7\%) da amostra selecionada. Constataseuma baixa produção científica relacionada ao tema, tendo sido publicado 10 artigos nos três últimos anos, compondo, assim, $71 \%$ das pesquisas encontradas no período selecionado. Quanto ao método 11 (79\%) dos estudos com abordagem descritivos ou qualitativos, 2 (14\%) com o delineamento estudo de ensaios clínicos randomizados e 1 (7\%) validação por meio de relatórios de expertise. Resultados esses que expressam a necessidade de maior rigor metodológico para garantir melhores evidências, uma vez, que a utilização do uso de simulação se traduz em muitos benefícios no processo de ensino e aprendizagem, principalmente no que tange nos aspectos atitudinais.

Observa-se no quadro 2, que 64\% (HERNANDES-PADILHA et al., 2016; LEWIS et al., 2016; DE OLIVEIRA COSTA et al., 2017; OLIVEIRA-KUMAKURA; SILVA; GONÇALVES, 2018; OLIVEIRA et al.,2018; JANICAS e NARCHI, 2019; MESQUITA; SANTANA; MAGRO, 2019; PADILHA et al., 2019; OLAUSSEN; 
HEGGDAL; TVED, 2020) dos estudos aplicaram a simulação enquanto estratégia de ensino e aprendizagem, 28\% (MAZZO et al., 2017; NEGRI et al., 2019; COGO et al., 2019; CANCINO et al., 2020) descrevem a construção de cenários simulados de alta fidelidade para o desenvolvimento de habilidade e competências, $8 \%$ utilizaram a simulação realística para identificar os preditores de escores mais altos de pensamento crítico (SHINNICK e WOO 2013).

Quadro 1: Caracterização dos artigos sobre estratégias de comunicação utilizadas com a criança hospitalizado, no períodode 2010-2020.

\begin{tabular}{|c|c|c|c|c|}
\hline Autor(es), Título & Ano & Periódico & Método & $\begin{array}{c}\text { Nível de } \\
\text { Evidência }\end{array}$ \\
\hline MA. Shinnick, MA. Woo & 2013 & $\begin{array}{c}\text { Nurse Education } \\
\text { Today }\end{array}$ & $\begin{array}{l}\text { Estudo Quase } \\
\text { experimental }\end{array}$ & $\begin{array}{l}\text { NE- } \\
\text { VI }\end{array}$ \\
\hline $\begin{array}{l}\text { Hernández PJM, Granero MJ, } \\
\text { Márquez HVV, Cortés RAE, } \\
\text { Fernández SC. }\end{array}$ & 2016 & $\begin{array}{c}\text { Acta Paulista de } \\
\text { Enfermagem }\end{array}$ & $\begin{array}{l}\text { Estudo Quase } \\
\text { experimental }\end{array}$ & $\begin{array}{l}\text { NE- } \\
\text { VI }\end{array}$ \\
\hline $\begin{array}{l}\text { Claire L, Joanne R, Zara M, } \\
\text { Rory I, Marian T. }\end{array}$ & 2016 & $\begin{array}{c}\text { BMC Palliative } \\
\text { Care }\end{array}$ & $\begin{array}{l}\text { Estudo Quase } \\
\text { experimental }\end{array}$ & $\begin{array}{l}\text { NE- } \\
\text { VI }\end{array}$ \\
\hline $\begin{array}{l}\text { Raphael ROC, Soraya MM, José } \\
\text { CAM, Marcelly SC, Marília AS. }\end{array}$ & 2017 & Revista Cuidarte & $\begin{array}{c}\text { Estudo Quanti- } \\
\text { Qualitativo }\end{array}$ & $\begin{array}{l}\text { NE- } \\
\text { VI }\end{array}$ \\
\hline $\begin{array}{l}\text { Oliveira KARS, Silva JLG, } \\
\text { Gonçalves N }\end{array}$ & 2018 & $\begin{array}{c}\text { Escola Anna } \\
\text { Nery }\end{array}$ & $\begin{array}{c}\text { Estudo Relato } \\
\text { de Caso }\end{array}$ & $\begin{array}{l}\text { NE- } \\
\text { VI }\end{array}$ \\
\hline $\begin{array}{l}\text { Saionara NO, Aline M, Jussara } \\
\text { GM, Jeferson R. }\end{array}$ & 2018 & $\begin{array}{c}\text { Revista Brasileira } \\
\text { de Enfermagem } \\
\text { REBEn }\end{array}$ & $\begin{array}{l}\text { Estudo Relato } \\
\text { de experiência }\end{array}$ & $\begin{array}{l}\text { NE- } \\
\text { VI }\end{array}$ \\
\hline $\begin{array}{l}\text { Mazzo A, Miranda FBG, Meska } \\
\text { MHG, Bianchini A, Bernardes } \\
\text { RM, Pereira Junior GA }\end{array}$ & 2018 & $\begin{array}{c}\text { Escola Anna } \\
\text { Nery }\end{array}$ & $\begin{array}{l}\text { Estudo Relato } \\
\text { de experiência }\end{array}$ & $\begin{array}{l}\text { NE- } \\
\text { VI }\end{array}$ \\
\hline Janicas RCSV, Narchi NZ. & 2019 & $\begin{array}{l}\text { Revista Latino } \\
\text { Americana de } \\
\text { Enfermagem }\end{array}$ & $\begin{array}{c}\text { Estudo } \\
\text { Randomizado }\end{array}$ & NE-II \\
\hline Autor(es), Título & Ano & Periódico & Método & Nível de \\
\hline
\end{tabular}




\begin{tabular}{|c|c|c|c|c|}
\hline & & & & Evidência \\
\hline $\begin{array}{l}\text { Elaine CN, Gerson APJ, Cezar } \\
\text { KCF, Juliana CF, Alessandra M. }\end{array}$ & 2019 & $\begin{array}{c}\text { Texto \& } \\
\text { Contexto } \\
\text { Enfermagem }\end{array}$ & $\begin{array}{c}\text { Estudo } \\
\text { descritivo de } \\
\text { Construção e } \\
\text { Validação }\end{array}$ & $\begin{array}{l}\text { NE- } \\
\text { VII }\end{array}$ \\
\hline $\begin{array}{l}\text { Ana LPC, Elisabeth FSL, } \\
\text { Fernanda RIP, Giovana EF, } \\
\text { Maria RRS. }\end{array}$ & 2019 & $\begin{array}{l}\text { Revista Gaúcha } \\
\text { de Enfermagem }\end{array}$ & $\begin{array}{l}\text { Estudo Relato } \\
\text { de Experiência }\end{array}$ & $\begin{array}{l}\text { NE- } \\
\text { VI }\end{array}$ \\
\hline $\begin{array}{l}\text { Mesquita HCT, Santana BS, } \\
\text { Magro MCS }\end{array}$ & 2019 & $\begin{array}{c}\text { Escola Anna } \\
\text { Nery }\end{array}$ & $\begin{array}{l}\text { Estudo Quase } \\
\text { experimental }\end{array}$ & $\begin{array}{l}\text { NE- } \\
\text { VI }\end{array}$ \\
\hline $\begin{array}{l}\text { José MP, Paulo PM, Ana R, José } \\
\text { R, Patrício C. }\end{array}$ & 2019 & \begin{tabular}{|c|} 
Journal Of \\
Medical Research
\end{tabular} & $\begin{array}{c}\text { Estudo } \\
\text { Randomizado }\end{array}$ & NE-II \\
\hline $\begin{array}{l}\text { Cancino KD, Arias M, Caballero } \\
\text { E, Escudero E. }\end{array}$ & 2020 & $\begin{array}{l}\text { Revista Latino } \\
\text { Americana de } \\
\text { Enfermagem }\end{array}$ & $\begin{array}{c}\text { Estudo } \\
\text { Quantitativo }\end{array}$ & $\begin{array}{l}\text { NE- } \\
\text { VI }\end{array}$ \\
\hline $\begin{array}{l}\text { Camilla O, Kristin H, Christine } \\
\text { RT. }\end{array}$ & 2020 & Nursing Open & $\begin{array}{c}\text { Estudo } \\
\text { Quantitativo }\end{array}$ & $\begin{array}{l}\text { NE- } \\
\text { VI }\end{array}$ \\
\hline
\end{tabular}

Quadro 2. Caracterização de objetivos e conclusões dos artigos sobre o "Uso Da Simulação Realística Como Estratégia de Ensino Paraos Cursos de Graduação em Enfermagem", publicados no período de 2010 a 2020.

\begin{tabular}{|c|c|c|}
\hline Autor (es) & Objetivo & Conclusão \\
\hline $\begin{array}{l}\text { MA. Shinnick, } \\
\text { MA. Woo. } \\
(2013)\end{array}$ & $\begin{array}{l}\text { Identificar os preditores de } \\
\text { escores maisl altos de } \\
\text { pensamento crítico }\end{array}$ & $\begin{array}{l}\text { Este estudo revela que o ganho de } \\
\text { conhecimento com a Simulação de Paciente } \\
\text { Humano não equivale a mudanças no } \\
\text { pensamento crítico.Ele expõe as variáveis da } \\
\text { idade avançada, maior conhecimento básico } \\
\text { e baixa autoeficácia em "gerenciar os níveis } \\
\text { de fluidos de um paciente" como preditores } \\
\text { de maior capacidade de pensamento crítico. }\end{array}$ \\
\hline
\end{tabular}




\begin{tabular}{|c|c|c|}
\hline s) & $\mathbf{0}$ & ão \\
\hline $\begin{array}{l}\text { Hernández } \\
\text { PJM, Granero } \\
\text { MJ, Márquez } \\
\text { HVV, Cortés } \\
\text { RAE, } \\
\text { Fernández SC. } \\
\text { (2016) }\end{array}$ & $\begin{array}{l}\text { Avaliar se um workshop de } \\
\text { simulação e curta duração } \\
\text { sobre punção da artéria radial } \\
\text { melhoraria a competência de } \\
\text { alunos de enfermagem em um } \\
\text { nível em que pudessem } \\
\text { praticar o procedimento em } \\
\text { um paciente vivo sem } \\
\text { comprometer sua segurança. }\end{array}$ & $\begin{array}{l}\text { O treinamento efetivo em punção arterial } \\
\text { baseado em simulação para estudantes de } \\
\text { enfermagem não necessariamente precisa } \\
\text { ser intensivo em recursos. Sessões de } \\
\text { treinamento bem planejadas e baseadas em } \\
\text { evidências, com uso de simuladores de baixa } \\
\text { tecnologia podem ajudar os educadores a } \\
\text { alcançarem bons resultados educacionais e } \\
\text { promover a segurança do paciente. }\end{array}$ \\
\hline $\begin{array}{lr}\text { Claire } & \text { L, } \\
\text { Joanne R,Zara } \\
\text { M, Rory I, } \\
\text { Marian T. } \\
(2016)\end{array}$ & $\begin{array}{l}\text { Avaliar o impacto de uma } \\
\text { intervenção simulada nas } \\
\text { atitudes dos estudantes de } \\
\text { graduação em enfermagem e } \\
\text { medicina em relação aos } \\
\text { cuidados em fim de vida. }\end{array}$ & $\begin{array}{l}\text { O aprendizado ativo e experimental, na forma } \\
\text { de ensino de simulação,ajuda a melhorar as } \\
\text { atitudes dos estudantes de graduação em } \\
\text { enfermagem e medicina no final da vida. Na } \\
\text { ausência de exposição clínica, a simulação é } \\
\text { uma alternativa viável para ajudar a preparar } \\
\text { osalunos para o seu papel profissional em } \\
\text { relação aos cuidados no final da vida. }\end{array}$ \\
\hline $\begin{array}{l}\text { Raphael } \\
\text { Soraya } \\
\text { José } \quad \text { CAM, } \\
\text { Marcelly SC, } \\
\text { Marília AS. } \\
\text { (2017) }\end{array}$ & $\begin{array}{l}\text { Identificar a percepção de } \\
\text { estudantes da graduação em } \\
\text { Enfermagem sobre a } \\
\text { simulação realística enquanto } \\
\text { estratégia de ensino e } \\
\text { aprendizagem. }\end{array}$ & $\begin{array}{l}\text { A simulação apresenta-se como uma } \\
\text { possibilidade viável e potencialmente } \\
\text { significativa no contexto do ensino de } \\
\text { graduação em Enfermagem }\end{array}$ \\
\hline $\begin{array}{l}\text { Oliveira } \\
\text { KARS, Silva } \\
\text { JLG, } \\
\text { Gonçalves N. } \\
(2018)\end{array}$ & $\begin{array}{l}\text { Relatar a experiência da } \\
\text { aplicação de diferentes } \\
\text { estratégias de ensino para } \\
\text { estudantes do Curso de } \\
\text { Graduação em Enfermagem } \\
\text { sobre o atendimento à vítima } \\
\text { de queimadura. }\end{array}$ & $\begin{array}{l}\text { É importante a incorporação de diferentes } \\
\text { estratégias de ensino ativo,como práticas de } \\
\text { simulação clínica, e-learning, aula } \\
\text { dialogada, estudos de caso, entre outras, } \\
\text { para o ensino na graduação em enfermagem } \\
\text { no cuidado à vítima de queimadura. }\end{array}$ \\
\hline
\end{tabular}




\begin{tabular}{|c|c|c|}
\hline Autor (res) & Objetivo & Conclusão \\
\hline $\begin{array}{lr}\text { Saionara } & \mathrm{NO}, \\
\text { Aline } & \mathrm{M}, \\
\text { Jussara } & \mathrm{GM}, \\
\text { Jeferson } & \mathrm{R} . \\
(2018) & \end{array}$ & $\begin{array}{l}\text { Relatar a experiência da } \\
\text { operacionalização da } \\
\text { simulação clínica como } \\
\text { estratégia pedagógica em uma } \\
\text { disciplina de um curso de } \\
\text { graduação em Enfermagem. }\end{array}$ & $\begin{array}{l}\text { A implantação da simulação clínica como } \\
\text { estratégia peda- gógica mostrou-se um } \\
\text { processo complexo, que precisa estar aberto } \\
\text { a sugestões e adaptações, permitindo que se } \\
\text { encontre o melhor meio deoperacionalizar a } \\
\text { logística deste processo, para que os } \\
\text { objetivos esperados sejam alcançados. O uso } \\
\text { de "pacientes simulados" para trabalhar as } \\
\text { compe- tências de comunicação, escuta e } \\
\text { percepção do real problema apresentado } \\
\text { pelo paciente, mostrou-se altamente efetivo, } \\
\text { assim como a opção por realizar o debriefing } \\
\text { coletivo com um grupo de estudantes, } \\
\text { estimulando-os a discutir os aspectos que } \\
\text { podemser continuados e os que precisam ser } \\
\text { revistos e repensados durante o atendimento } \\
\text { ao paciente. }\end{array}$ \\
\hline $\begin{array}{l}\text { Mazzo A, } \\
\text { Miranda FBG, } \\
\text { Meska MHG, } \\
\text { Bianchini A, } \\
\text { Bernardes RM, } \\
\text { Pereira Junior } \\
\text { GA. (2018) }\end{array}$ & $\begin{array}{l}\text { Descrever a construção de } \\
\text { cenário clínico simulado de } \\
\text { alta fidelidade "Assistência de } \\
\text { enfermagem ao paciente com } \\
\text { lesão por pressão. }\end{array}$ & $\begin{array}{l}\text { A construção de cenários clínicos simulados } \\
\text { planejados, estruturados e validados, } \\
\text { aproxima o aprendiz da prática clínica } \\
\text { possibilitando o desenvolvimento de } \\
\text { competências necessárias para a prevenção e } \\
\text { tratamento de lesão por pressão. Implicações } \\
\text { para a prática: em cenários clínicos } \\
\text { simulados bem delineados aproximam com } \\
\text { fidelidade o treino da assistência de } \\
\text { enfermagem ao paciente com lesão por } \\
\text { pressão }\end{array}$ \\
\hline
\end{tabular}




\begin{tabular}{|c|c|c|}
\hline Aut & bjjetivo & Conclusão \\
\hline $\begin{array}{l}\text { Janicas } \\
\text { RCSV, } \\
\text { Narchi NZ. } \\
(2019)\end{array}$ & $\begin{array}{l}\text { Comparar o desempenho } \\
\text { clínico de discentes de } \\
\text { Enfermagem em cenários } \\
\text { de aprendizagem com e sem } \\
\text { debriefing em um centro de } \\
\text { simulação. }\end{array}$ & $\begin{array}{l}\text { Os resultados desta investigação } \\
\text { mostram que os estudantes do grupo } \\
\text { experimental apresentaram melhores } \\
\text { resultados de aprendizagem quando } \\
\text { comparados ao grupo-controle, o que } \\
\text { fortaleceu a evidência deque o uso de } \\
\text { cenários com debriefing realmente foi } \\
\text { eficaz no aprendizado, corroborando a } \\
\text { hipótese de que essa técnica de ensino } \\
\text { melhora o desempenho clínico na } \\
\text { assistência de enfermagem }\end{array}$ \\
\hline $\begin{array}{l}\text { Elaine } \mathrm{CN} \text {, } \\
\text { Gerson } \mathrm{APJ}, \\
\text { Cezar } \mathrm{KCF}, \\
\text { Juliana } \mathrm{CF}, \\
\text { Alessandra } \\
\text { M. (2019) }\end{array}$ & $\begin{array}{l}\text { Construir e validar um } \\
\text { cenário de simulação } \\
\text { clínica de alta fidelidade } \\
\text { sobre assistência de } \\
\text { enfermagem a pacientes } \\
\text { com colostomia. }\end{array}$ & $\begin{array}{l}\text { Os resultados mostram que para práticas } \\
\text { simuladas bem delineadas eexitosas são } \\
\text { necessárias a elaboração criteriosa, a } \\
\text { validação e a testagem prévia das } \\
\text { atividades planejadas. Nas práticas } \\
\text { destinadas à caracterização do paciente } \\
\text { colostomizado, o uso de técnicas } \\
\text { específicas de maquiagem são efetivas } \\
\text { e conferem realismo à atividade. }\end{array}$ \\
\hline $\begin{array}{l}\text { Ana LPC, } \\
\text { Elisabeth } \\
\text { FSL, } \\
\text { Fernanda } \\
\text { RIP, Giovana } \\
\text { EF, Maria } \\
\text { RRS. (2019) }\end{array}$ & $\begin{array}{l}\text { Descrever a construção de } \\
\text { cenários } \\
\text { desenvolvimento da técnica } \\
\text { de simulação realística em } \\
\text { saúde sobre administração } \\
\text { segura de medicamentos } \\
\text { pela enfermagem. }\end{array}$ & $\begin{array}{l}\text { A simulação permitiu a reflexão do } \\
\text { "modo de fazer" das equipes, ajustando } \\
\text { o processo de preparo e administração de } \\
\text { medicamentos às recomendações } \\
\text { institucionais com foco na segurança do } \\
\text { processo. }\end{array}$ \\
\hline
\end{tabular}




\begin{tabular}{|c|c|c|}
\hline Aut & bjetivo & Conclusão \\
\hline $\begin{array}{l}\text { Mesquita } \\
\text { HCT, } \\
\text { Santana BS, } \\
\text { Magro MCS. } \\
(2019)\end{array}$ & $\begin{array}{l}\text { Comparar o efeito do } \\
\text { emprego da estratégia de } \\
\text { simulação combinada à } \\
\text { teoria com a simulação, per } \\
\text { se, no ganho de } \\
\text { autoconfiança } \\
\text { profissionais } \\
\text { enfermagem durante o } \\
\text { manejo do paciente em } \\
\text { parada cardiopulmonar. }\end{array}$ & $\begin{array}{l}\text { nível de autoconfiança dos profissionais } \\
\text { de enfermagem para atendimento ao } \\
\text { paciente em parada cardiopulmonar } \\
\text { melhorou significativamente após } \\
\text { emprego da simulação combinada a } \\
\text { teoria. }\end{array}$ \\
\hline $\begin{array}{ll}\text { José } & \text { MP, } \\
\text { Paulo PM, } & \text { Ana R, José } \\
\text { R, Patrício C. } \\
(2019)\end{array}$ & 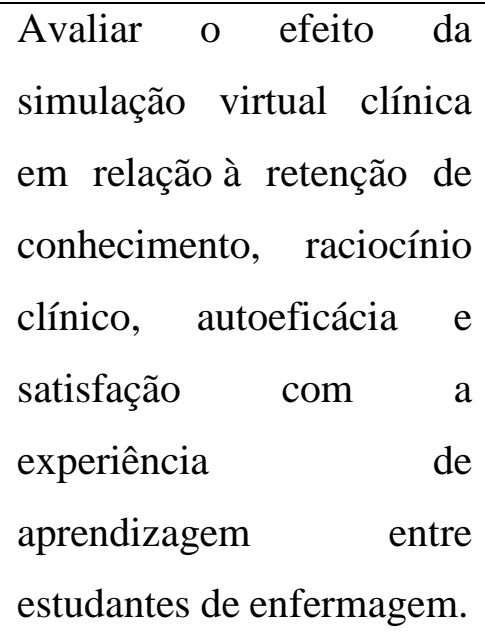 & $\begin{array}{l}\mathrm{O} \text { grupo experimental fez melhorias } \\
\text { mais significativas no conhecimento } \\
\text { após a intervenção }(\mathrm{P}=0,001 ; \mathrm{d}=1,13) \\
\text { e } 2 \text { meses depois }(\mathrm{P}=0,02 ; \mathrm{d}=0,75) \text {, } \\
\text { além de apresentar níveis mais altos de } \\
\text { satisfação com a aprendizagem }(\mathrm{P}<.001 \text {; } \\
\mathrm{d}=1,33) \text {. Não encontramos diferenças } \\
\text { estatísticas nas percepções } \\
\text { autoeficácia }(\mathrm{P}=0,9 ; \mathrm{d}=0,054) .\end{array}$ \\
\hline $\begin{array}{lr}\text { Cancino } & \mathrm{KD}, \\
\text { Arias } & \mathrm{M}, \\
\text { Caballero } & \mathrm{E}, \\
\text { Escudero } & \mathrm{E} . \\
(2020) & \end{array}$ & $\begin{array}{l}\text { Determinar a validade de } \\
\text { face e conteúdo de um } \\
\text { roteiro de avaliação de } \\
\text { administração segura de } \\
\text { medicamentos em } \\
\text { estudantes de enfermagem. }\end{array}$ & $\begin{array}{l}\text { O roteiro se encontra em condições de } \\
\text { ser testado para avaliar a administração } \\
\text { segura de medicamentos em estudantes } \\
\text { de enfermagem, principalmente em } \\
\text { ambiente de simulação, como uma } \\
\text { ferramenta para o corpo acadêmico. }\end{array}$ \\
\hline
\end{tabular}




\begin{tabular}{|c|c|c|}
\hline Autor (res) & \begin{tabular}{|l} 
Objetivo \\
\end{tabular} & Conclusão \\
\hline $\begin{array}{l}\text { Camilla O, Kristin, H, } \\
\text { Christine RT. (2020) }\end{array}$ & $\begin{array}{l}\text { Identificar elementos na } \\
\text { simulação baseada em } \\
\text { cenário associados à } \\
\text { satisfação dos estudantes } \\
\text { de enfermagem com a } \\
\text { atividade de simulação e } \\
\text { autoconfiança no } \\
\text { gerenciamento da situação } \\
\text { simuladado paciente. }\end{array}$ & $\begin{array}{l}\text { Os escores médios de } \\
\text { autoconfiança e satisfação } \\
\text { foram } 4,16 \text { e } 4,57, \\
\text { respectivamente. } \mathrm{Na} \\
\text { análise final de regressão } \\
\text { linear múltipla, o } \\
\text { aprendizado ativo foi } \\
\text { associado à satisfação com } \\
\text { a atividade de simulação, } \\
\text { enquanto objetivos claros e } \\
\text { o aprendizado ativo foram } \\
\text { associados à autoconfiança } \\
\text { no gerenciamento da } \\
\text { situação simulada do } \\
\text { paciente. }\end{array}$ \\
\hline
\end{tabular}




\section{DISCUSSÃO}

Este estudo buscou analisar a prática clínica simulada nos cursos de graduação em enfermagem como estratégia de ensino e aprendizagem. As evidências refletem que o uso da simulação realista como estratégia de ensino tem sido de interesse nacional e internacional , aproximando os estudantes e profissionais aos contextos reais simulados, oferecendo completa segurança para os envolvidos, buscando desenvolver não somente habilidades técnicas, como também o gerenciamento de crises, liderança, trabalho em equipe, raciocínio clínico em situações críticas ou que possam provocar prejuízos ao paciente real, sendo uma ferramenta poderosa de aprendizagem (JANICAS e NARCHI, 2019).

Está metodologia nos cursos de Enfermagem, é uma realidade em Portugal e tem contribuído para a melhoria do processo formativo e o desenvolvimento de autoconfiança,autonomia e satisfação dos estudantes. No Brasil, os estudos sobre a temática ainda são incipientes e carece de produção de evidências (DE OLIVEIRA COSTA et al., 2017).

A literatura ressalta que para execução desta prática simulada os objetivos de ensino-aprendizagem devem ser definidos de acordo com os resultados almejados. Utilizando a Taxonomia de Bloom e sua classificação hierárquica dos objetivos de aprendizagem, os educadores, conscientemente, procuram meios de estimular nos seus estudantes, raciocínio e abstrações de alto nível, sem distanciar-se dos objetivos instrucionais previamente propostos, para o desenvolvimento de suas competências e habilidades, preconizadas pelas DCNs (MAZZO et al., 2017).

Um estudo realizado em um curso de graduação de enfermagem de uma universidade pública de Campinas-SP com a utilização de cenários de simulação, ressalta a necessidade de desenvolvimento das áreas cognitivas, afetivas e psicomotoras, ou seja, habilidadestécnicas e não técnica.

O Nurse Educator Competencies recomenda, para desenvolveressas áreas o uso de abordagens educacionais que reflitam a teoria e as práticas educacionais contemporâneas, neste contexto, sendo emprega as simulações (OLIVEIRAKUMAKURA; SILVA; GONÇALVES, 2018). A simulação clínica permite ao professor acompanhar o desenvolvimento do estudante, emitindo feedbacks 
construtivos que auxiliam no aperfeiçoamento e desenvolvimento de suas competências, o que vai ao encontro do que se propõe na avaliação formativa (OLIVEIRA et al., 2018).

Na Enfermagem, o ensino por meio da demonstração de técnicas e procedimentos deforma simulada não é algo novo ou recente, todavia, a simulação clínica possui uma proposta de operacionalização diferenciada, que busca instigar a reflexão do estudante a partir de seus atos e de seus conhecimentos, ultra-passando a repetição de ações demonstradas pelo professor, tornando-se assim o centro do processo de ensino que é apoiado e mediado pelo professor (OLIVEIRA et al., 2018; MESQUITA; SANTANA; MAGRO, 2019).

O raciocínio clínico em enfermagem é um elemento essencial do exercício profissionale dos cuidados qualificados de enfermagem, pois está presente nas ações e decisões assistenciais do enfermeiro, a simulação têm sido cada vez mais valorizadas no campo deformação profissional. O debriefing compreende a ultima etapa da simulação, é ummomento de reflexão da prática realizada em que os estudantes exploram as suas ações,emoções e os processos de pensamentos que influenciam sua tomada de decisão,desenvolvem a capacidade de autoavaliação, de criticar e de ouvir críticas, aprendendo apartir desta rica experiência. Nessa perspectiva, o debriefing tem sido identificado como uma das oportunidades mais privilegiadas de aprendizagem (JANICAS e NARCHI, 2019)

Um estudo realizado na Noruega envolvendo estudantes de enfermagem, apresentamem seus resultados que o aprendizado ativo por meio da simulação e debriefing contribuíram para satisfação e a autoconfiança dos estudantes. O debriefing tem emergidocomo uma estratégia extremamente importante para a aplicação eficaz da educação; representa o pilar central da simulação (OLAUSSEN; HEGGDAL; TVEDT, 2020).

Para o sucesso durante a simulação se faz necessário um roteiro que permite avaliaras competências e fornecer um feedback padronizado. O roteiro, ajuda a estruturar o processo, selando pela qualidade e segurança, tanto em atividades de simulação clínica quanto na prática clínica, configurando-se como roteiro padronizado para a prática docente (CANCINO et al., 2020). 
Os resultados de um estudo realizado por uma universidade privada de São Paulo, mostram que os estudantes do grupo experimental apresentaram melhores resultados de aprendizagem quando comparados ao grupo-controle, o que fortaleceu a evidência de que o uso de cenários com debriefing realmente foi eficaz no aprendizado, corroborando a hipótese de que essa técnica de ensino melhora o desempenho clínico na assistência de enfermagem. Esses resultados evidenciam que toda simulação baseada em experiência de aprendizagem deve incluir o planejamento de debriefing, componente valioso para produçãoe ganho de conhecimento (JANICAS e NARCHI, 2019).

Outro aspecto importante em relação aos cenários de simulação para assistência de enfermagem, é o fato da complexidade do cenário clínico simulado não estar relacionada à complexidade dos recursos utilizados e sim aos objetivos de aprendizagem determinados, os quais para serem bem-sucedidos precisam ser claros e permitir que os alunos se aproximem ao máximo da realidade para maior fidelidade (NEGRI et al., 2019; COGO et al., 2019; SHINNICK e WOO, 2013).

Uma pesquisa realizada na universidade do Sudeste da Espanha, corrobora quando menciona complexidade, tecnologia versus objetivos de aprendizagem bem estruturados. Neste estudo foi realizado treinamento baseado em simulação hibrida autodirigida com usode equipamentos de baixa tecnologia, concluindo que este tipo de tecnologia pode ajudar os educadores a alcançarem bons resultados educacionais e promove a prática segura (HERNANDEZ-PADILLA et al., 2016; SHINNICK e WOO, 2013).

Com tudo, cabe ressaltar que os estudantes do século XXI cresceram usando as tecnologias da informação e comunicação (TIC) no dia a dia. O uso das TIC leva a diferentes processos de aprendizagem e processos de estruturação da informação.

As inovações nas tecnologias de simulação em saúde levaram à simulação virtual, na escola de enfermagem do Porto, em Portugal por meio de um ensaio clínico randomizado identificou melhor retenção de conhecimento e raciocínio clínico, além de aumentar a satisfação com a experiência de aprendizagem entre os estudantes de enfermagem. A inclusão de tecnologia digital e virtual na educação em tempos de desafios no Brasil, irá contribuir para a recriação da realidade usando pacientes virtuais (PADILHA et al.,2019). 
$\mathrm{Na}$ educação, decidir e definir os objetivos de aprendizagem significa estruturar, de forma consciente, o processo educacional de modo a oportunizar mudanças de pensamentos, ações e condutas. A simulação é uma alternativa viável para ajudar a preparar os alunos para seu papel profissional em relação aos cuidados e também para adotar uma abordagem interprofessional (LEWIS et al., 2016).

\section{CONSIDERAÕES FINAIS}

O emprego da simulação realística nos cursos de enfermagem foi apontado como uma estratégia de ensino que favorece um melhor aprendizado, com o desenvolvimento de diversas habilidades e competências.

Os dados portanto, revelaram que os alunos identificam a prática da simulação realística como uma abordagem mais efetiva para o ensino-aprendizagem do que o ensino tradicional, estratégia pedagógica que permite uma maior vivência prática e realística, colaborando para segurança do paciente. Seguir um roteiro estruturado para simulação e debriefing vai ajudar os educadores e até mesmo os alunos a facilitar o ambiente de aprendizagem e melhorar o desenvolvimento do julgamento clínico.

A utilização desta metodologia é de grande importância na formação do graduando, já que ela desenvolve a formação do pensamento crítico, reflexão e estimula á pró-atividade sendo uma ferramenta integradora da teoria e da prática, permitindo ao aluno desenvolver a capacidade de resolver problemas.

Sugere-se a realização de outras pesquisas que expressem maior rigor metodológico para garantir melhores evidências, uma vez, que a utilização do uso de simulação se traduz em muitos benefícios no processo de ensino e aprendizagem, principalmente no que tange os aspectos atitudinais. 


\section{REFERÊNCIAS}

ARAUJO, U. A. M. Máscaras inteiriças Tukúna: possibilidades de estudo de artefatos de museu para o conhecimento do universo indígena. 1985. 102 f. Dissertação (Mestrado em Ciências Sociais) - Fundação Escola de Sociologia e Política de São Paulo, São Paulo, 1986.

BATISTA, Cássia Beatriz. Movimentos de reorientação da formação em saúde e as iniciativas ministeriais para as universidades. Barbaroi, n. 38, p. 97-125, 2013.

BURNS, Patricia B.; ROHRICH, Rod J.; CHUNG, Kevin C. The levels of evidence and their role in evidence-based medicine. Plastic and reconstructive surgery, v. 128, n. 1, p. 305, 2011.

CANCINO, Karen Domínguez et al. Elaboração de um roteiro de avaliação de administração segura de medicamentos em estudantes de enfermagem. Revista LatinoAmericana de Enfermagem, v. 28, 2020.

COGO, Ana Luísa Petersen et al. Construção e desenvolvimento de cenários de simulação realística sobre a administração segura de medicamentos. Revista Gaúcha de Enfermagem, v. 40, 2019.

DE OLIVEIRA COSTA, Raphael Raniere et al. A simulação no ensino de enfermagem: reflexões e justificativas a luz da bioética e dos direitos humanos. Acta Bioethica, v. 24, n. 1, p. 31-38, 2018.

DE OLIVEIRA COSTA, Raphael Raniere et al. Percepção de estudantes da graduação em enfermagem sobre a simulação realística. Revista Cuidarte, v. 8, n. 3, p. 1799-1808, 2017.

FERRAZ, Ana Paula do Carmo Marcheti; BELHOT, Renato Vairo. Taxonomia de Bloom: revisão teórica e apresentação das adequações do instrumento para definição de objetivos instrucionais. Gestão \& Produção, v. 17, p. 421-431, 2010.

FERREIRA, Márcia de Assunção. A comunicação no cuidado: uma questão fundamental na enfermagem. Revista Brasileira de Enfermagem, v. 59, p. 327-330, 2006.

FERREIRA, Raína Pleis Neves et al. Simulação realística como método de ensino no aprendizado de estudantes da área da saúde. Revista de Enfermagem do Centro-Oeste Mineiro, v. 8, 2018.

GALVÃO, Taís Freire; PANSANI, Thais de Souza Andrade; HARRAD, David. Principais itens para relatar Revisões sistemáticas e Meta-análises: A recomendação PRISMA. Epidemiologia e Serviços de Saúde, v. 24, p. 335-342, 2015.

GONZALEZ, Alberto Durán; ALMEIDA, Márcio José de. Ativação de mudanças na formação superior em saúde: dificuldades e estratégias. Revista Brasileira de Educação Médica, v. 34, p. 238-246, 2010. 
HERNÁNDEZ-PADILLA, José Manuel et al. Efeitos de um workshop de simulação sobre a competência em punção arterial de estudantes de enfermagem. Acta Paulista de Enfermagem, v. 29, p. 678-685, 2016.

JANICAS, Rita de Cassia Silva Vieira; NARCHI, Nádia Zanon. Evaluation of nursing students' learning using realistic scenarios with and without debriefing. Revista latinoamericana de enfermagem, v. 27, 2019.

KELLY, Susan Harrison. Evaluation methods used in simulation: A survey of faculty and student perceptions in an undergraduate nursing program. 2014. Tese de Doutorado. University of Pittsburgh.

LEWIS, Claire et al. The impact of a simulated intervention on attitudes of undergraduate nursing and medical students towards end of life care provision. BMC Palliative Care, v. 15, n. 1, p. 1-6, 2016.

MAZZO, Alessandra et al. Ensino de prevenção e tratamento de lesão por pressão utilizando simulação. Escola Anna Nery, v. 22, 2017.

MENDES, Karina Dal Sasso; SILVEIRA, Renata Cristina de Campos Pereira; GALVÃO, Cristina Maria. Revisão integrativa: método de pesquisa para a incorporação de evidências na saúde e na enfermagem. Texto \& contexto-enfermagem, v. 17, p. 758$764,2008$.

MESQUITA, Hanna Clara Teixeira; SANTANA, Breno de Sousa; MAGRO, Marcia Cristina da Silva. Efeito da simulação realística combinada à teoria na autoconfiança e satisfação de profissionais de enfermagem. Escola Anna Nery, v. 23, 2019.

NEGRI, Elaine Cristina et al. Clinical simulation with dramatization: gains perceived by students and health professionals. Revista latino-americana de enfermagem, v. 25 , 2017.

NEGRI, Elaine Cristina et al. Construção e validação de cenário simulado para assistência de enfermagem a pacientes com colostomia. Texto \& Contexto-Enfermagem, v. 28, 2019.

OLAUSSEN, Camilla; HEGGDAL, Kristin; TVEDT, Christine Raaen. Elements in scenario-based simulation associated with nursing students' self-confidence and satisfaction: A cross-sectional study. Nursing open, v. 7, n. 1, p. 170-179, 2020.

OLIVEIRA, Saionara Nunes de et al. Da teoria à prática, operacionalizando a simulação clínica no ensino de Enfermagem. Revista Brasileira de Enfermagem, v. 71, p. 1791$1798,2018$.

OLIVEIRA-KUMAKURA, Ana Railka de Souza; SILVA, Juliany Lino Gomes; GONÇALVES, Natália. Da aula teórica ao uso da simulação para ensinar o cuidar de pessoas com queimaduras: relato de caso. Escola Anna Nery, v. 22, 2018. 
PADILHA, José Miguel et al. Clinical virtual simulation in nursing education: randomized controlled trial. Journal of medical Internet research, v. 21, n. 3, p. e11529, 2019..

SANTOS, Cristina Mamédio da Costa; PIMENTA, Cibele Andrucioli de Mattos; NOBRE, Moacyr Roberto Cuce. A estratégia PICO para a construção da pergunta de pesquisa e busca de evidências. Revista Latino-Americana de Enfermagem, v. 15, p. 508-511, 2007.

SHINNICK, Mary Ann; WOO, Mary A. The effect of human patient simulation on critical thinking and its predictors in prelicensure nursing students. Nurse Education Today, v. 33, n. 9, p. 1062-1067, 2013.

SOUZA, Josiane et al. Debriefing como ferramenta de avaliação qualitativa no ensino simulado. CIAIQ 2017, v. 2, 2017.

TIEPPO, Carla; FONSECA, Rochele Paz. Como as pessoas aprendem?. A moderna Educação. PUCRS. E-book (não paginado). Disponível em:<http://ebooks.pucrs.br/edipucrs/ame/como-aspessoasaprendem/assets/download/livro-offline.pdf. Acessado em 15 de mar. de 2020.

TUNES, Elizabeth; TACCA, Maria Carmen VR; BARTHOLO JÚNIOR, Roberto dos Santos. O professor e o ato de ensinar. Cadernos de pesquisa, v. 35, p. 689-698, 2005.

VILELLA, Denise Santos; LEITE, Leia Magna; NASSAR, Maria Elisa Diniz. A simulação realística como estratégia de ensino em atendimento pré-hospitalar: um relato de experiência. São Paulo (SP): Prefeitura de São Paulo, 2010. 